\title{
CONCEITOS E CARACTERÍSTICAS DE SISTEMAS POKA-YOKES: UMA REVISÃO DE LITERATURA
}

\section{CONCEPTS AND FEATURES ABOUT POKA-YOKE SYSTEMS: A LITERATURE REVIEW}

\author{
Gabriel Vidor *E-mail: gvidor@producao.ufrgs.br \\ Tarcísio Abreu Saurin* E-mail: saurin@ufrgs.br \\ *Universidade Federal do Rio Grande do Sul, Porto Alegre, RS
}

\begin{abstract}
Resumo: O objetivo deste trabalho é realizar uma análise sobre métodos, técnicas e diretrizes para projeto, operação e manutenção de sistemas poka-yokes. Paralelamente pretende-se contribuir com a apresentação do conceito de poka-yoke e quais as classificações a ele relativas. Dessa forma, conduziu-se uma revisão bibliográfica sobre conceitos de poka-yokes, classificação de sistemas poka-yokes, tipos de inspeção, tipos de erro, a relação da estabilidade e controle estatístico de processo com sistemas de prevenção de erro, técnicas e diretrizes existentes para projetar e gerir poka-yokes. Para cada tema realizou-se uma análise, estabelecendo a relação com os poka-yokes. Ao fim do trabalho constatou-se a ausência de um sistema para gestão de poka-yoke (SGPK), decorrente das inconsistências das técnicas existentes para projeto, operação e manutenção de poka-yoke.
\end{abstract}

Palavras-chave: Qualidade. Manutenção. Poka-yoke. Inspeção. Erro.

\begin{abstract}
This paper has the purpose of analyzing methods, techniques and guidelines for the design, operation and maintenance of poka-yoke systems. In parallel it intends to contribute with concepts about what poka-yoke are and their classification. Therefore a literature review was made about systems poka-yoke concepts, poka-yoke systems classification, kinds of inspections, kinds of mistakes, the relationship between stability and statistical process control with mistake proofing systems, techniques and directions to design and manage poka-yoke. An analysis was carried out with each subject relating poka-yoke with the subject. At the end the absence of a poka-yoke management system was evident, because techniques for design, operation and maintenance aren't consistent.
\end{abstract}

Keywords: Quality. Maintenance. Poka-yoke. Inspection. Mistake.

\section{INTRODUÇÃO}

A falta de estabilidade nos sistemas de manufatura, relatada por Liker e Meier (2007), é uma das principais causas de perdas e uma barreira para a 
implantação do fluxo contínuo, uma das metas dos sistemas de produção enxuta (PE). Dentre as estratégias para estabilização, os poka-yokes têm despertado crescente interesse na indústria e na academia, em função da aparente simplicidade de implantação e caráter intuitivo de seu funcionamento. De fato, Grout (2007) e Formoso et al. (2002) relatam a aplicação de poka-yoke em ambientes diversos, tais como construção civil, indústria automotiva, metalúrgica, saúde, logística, entre outras.

O termo poka-yoke tem sua origem nas experiências da Toyota Motors Company, que visavam obter zero defeitos na produção e eliminar as inspeções de qualidade. Os métodos para atingir tal objetivo foram inicialmente chamados de "à prova de bobos (baka-yokes)", sendo que posteriormente reconheceu-se que isso era ofensivo aos trabalhadores e a denominação mudou para "à prova de erros" ou "livres de falhas" (poka-yoke). Inicialmente o objetivo era prevenir o erro humano no trabalho, visto como a principal causa dos defeitos (SHIMBUN, 1988).

Contudo, a expressão poka-yoke ou à prova de erros ou falhas é pouco precisa, havendo desde estudos que entendem que os poka-yokes são limitados aos dispositivos físicos que controlam defeitos (BENDELL et al., 1995) até estudos com uma visão abrangente, que entendem os mesmos como sistemas de garantia de qualidade e redução de variabilidade (MCGEE, 2005). Além disso, os métodos para projeto, operação e manutenção de poka-yokes estão desvinculados dos conceitos de estabilidade estatística de processo. Tal vínculo deveria existir, pois o controle estatístico da qualidade permite a identificação da frequência das causas aleatórias de um determinado processo (MONTGOMERY, 2004), o que constitui um grupo de informações para potenciais desenvolvimentos e implementações de sistemas pokayokes. Contudo, vale ressaltar que um dos motivos pelos quais os poka-yokes foram disseminados na Toyota foi justamente a tentativa de reduzir a dependência do controle estatístico da qualidade, visto que esse, por definição, aceita margens de erro que são incompatíveis com a meta de zero defeitos (SHINGO, 2000). Entretanto, tal argumento possui limitações, tais como: (a) os poka-yokes não podem substituir o controle estatístico de processo em 100\% dos casos, seja pela impossibilidade técnica de projetar o poka-yoke ou pela natureza da característica de qualidade a ser inspecionada (por exemplo, resistência mecânica de componentes, 
cuja verificação pode exigir ensaios destrutivos); (b) os poka-yokes também são sujeitos a falhas, uma vez que freqüentemente são constituídos por componentes com confiabilidade inferior a 100\% (por exemplo, sensores); (c) conforme já comentado, o controle estatístico pode subsidiar o projeto de poka-yokes, apontando onde os mesmos são prioritários.

Portanto, o objetivo desse trabalho é analisar um conjunto de classificações e conceitos de poka-yokes identificados na literatura, tendo em vista a proposição de diretrizes de projeto, operação, manutenção e descontinuidade de uso dos pokayokes. Para tanto, o trabalho contextualiza o papel dos poka-yokes no controle de qualidade, enfatizando sua contribuição nas operações de inspeção. Em seguida, são apresentadas as diretrizes encontradas na literatura para projeto, operação e manutenção de poka-yokes no contexto do controle de qualidade.

\section{OS MECANISMOS DE CONTROLE DE QUALIDADE}

Devido à diversidade de elementos existentes para controlar e gerir a qualidade, neste trabalho são discutidos os poka-yokes e as operações de inspeção, haja vista o objetivo traçado para o estudo. Contudo, é necessário também uma discussão em relação ao conceito de controle da qualidade, dada a abrangência desse tema e sua importância para fundamentar uma discussão sobre sistemas poka-yokes.

\subsection{Definição de controle de qualidade}

Conforme Garvin (1992) a qualidade é uma disciplina em formação, podendo ser iniciada com os programas de inspeção, passando pelo controle estatístico da qualidade e garantia, até a fase de gestão estratégica. Nesse sentido a definição conceitual de qualidade é vinculada ao seu contexto. Conforme define Crosby (1999) a qualidade é o atendimento de requisitos dos clientes, portanto não é necessariamente sinônimo de virtude, brilho, luxo ou peso, nem tão pouco é intangível e impossível de ser mensurada. 
Similarmente o controle da qualidade é na sua essência "o processo regulador por meio do qual se mede o desempenho real da qualidade, comparandoo com os objetivos da qualidade e agindo-se sobre a diferença" (JURAN et al., 1991). Observam-se neste conceito questões implícitas como as inspeções do produto e a existência de um departamento da qualidade. Juran (1991) introduziu o termo controle da qualidade no início do século XX como sinônimo de prevenção de defeitos. Entretanto, em torno de 1940 houve uma propagação do conceito de controle estatístico de processo (CEP), restringindo o conceito de controle de qualidade à engenharia da qualidade, que acabou por deturpar a idéia original, de gestão organizacional, do controle da qualidade.

Posteriormente, Feigenbaum (1994) introduziu o termo “Total Quality Control (TQC)", considerando o controle de qualidade como um sistema que integra esforços para desenvolvimento, manutenção e aperfeiçoamento da qualidade de vários processos e produtos em uma organização, de forma a permitir a satisfação do cliente.

Decorrente de sua abrangência o controle de qualidade requer uma mudança cultural da organização, com o comprometimento de todas as pessoas (ISHIKAWA, 1993). Essa mudança, como propõe Deming (1990), está baseada em quatorze princípios de controle de qualidade, que envolvem fatores como liderança, aprendizagem, melhoria contínua e comprometimento.

A sistematização do controle de qualidade desenvolve-se através de ferramentas e técnicas da engenharia da qualidade e pela gestão da qualidade. Esses dois tipos de controle de qualidade classificam as ferramentas, associando-as a métodos com base estatística, ou relacionando-as às técnicas de gestão. Nesse segundo grupo enquadram-se elementos como poka-yokes e operações de inspeções, que podem ser aplicadas a operações, processos e a combinação de processos e operações. As técnicas podem ser combinadas, a fim de incrementar as análises. Nesse trabalho discutem-se técnicas aplicadas a operações, a processos e a combinação de processos e operações, a fim de subsidiar o objetivo proposto. 


\subsection{Conceitos de inspeção}

A inspeção consiste da comparação do produto com os requisitos aplicáveis a esse produto (HIRATA, 1993). Dessa forma, qualquer diferença entre estes requisitos e o resultado da inspeção pode ser considerada uma anormalidade. De fato, Shingo (1988) ressalta que as inspeções podem ser classificadas de acordo com o seu objetivo, que pode ser descobrir defeitos, reduzir defeitos ou eliminar defeitos. As classes propostas por Shingo são:

a) a inspeção por julgamento que tem a característica de descobrir defeitos, sendo aplicada aos produtos de forma a julgá-los defeituosos ou nãodefeituosos, garantindo que o produto defeituoso não chegue a clientes internos ou externos. De acordo com Ghinato (1996) este tipo de inspeção normalmente é aplicado a lotes inteiros de produção, posteriormente ao seu processamento ou em estágios finais de processo, o que não evita a produção de produtos defeituosos;

b) a inspeção informativa que tem como objetivo reduzir defeitos, na medida em que há feedback acerca dos defeitos identificados para o responsável pelo processo (GHINATO, 1996). Esse método, na visão Shingo (1988), é superior à inspeção por julgamento, contudo é ineficaz para a obtenção do zero defeito, visto que a ênfase está na detecção de defeitos no produto, ao invés da detecção de erros no processamento. Shingo (1988) classifica esse método em três categorias:

- o Controle Estatístico de Processo (CEP), bem como as cartas de controle, de acordo com Dias e Infante (2008), introduzidas por Shewhart em 1931. Prajapati e Mahapatra (2008) acrescentam que desde a criação das técnicas por Shewhart outras técnicas além das cartas de controle foram desenvolvidas e aplicadas no CEP, como as sete ferramentas da qualidade, assim denominadas por Montgomery (2004). Além disso, essas cartas de controle são vistas como poderosas ferramentas para detectar mudanças em processos produtivos ou em parâmetros dos processos produtivos (DIAS e INFANTE, 2008; WANG e ZHANG, 2008). Conforme Montgomery 
(2004) em qualquer processo de produção, independente de quão bem planejado ou mantido ele seja, sempre existirá variabilidade. Essa variabilidade é um efeito de muitas pequenas causas, essencialmente inevitáveis. Dentro do CEP essa variabilidade é entendida como "sistema estável de causas aleatórias" (MONTGOMERY, 2004), portanto quando um processo opera apenas com causas aleatórias de variação, também definidas como causas comuns por Shewhart (1931), ele está sob controle estatístico (as causas aleatórias são inerentes ao processo). Além dessas causas aleatórias existem também no controle de processo as causas atribuíveis. Uma das condições para um processo operar fora de controle é quando as causas atribuíveis estão fora dos limites de controle (DIAS e INFANTE, 2008). Uma causa atribuível é considerada fora dos limites quando assumir um valor superior ou inferior da média acrescido ou decrescido, respectivamente de três desvios padrão. Para Montgomery (2004) processos operam sob controle durante longos períodos de tempo. No entanto, causas atribuíveis normalmente ocorrem de maneira aleatória, resultando em um deslocamento para fora do estado de controle (instabilidade do processo), onde uma maior proporção da saída de processo não corresponde às exigências, ou seja, uma maior parte do que está sendo gerado estará fora dos limites de especificação (LSE - limite superior de especificação, LIE - limite inferior de especificação). Montgomery (2004) afirma que o objetivo maior do controle estatístico de processo é detectar rapidamente a ocorrência da instabilidade de processo (causas atribuíveis da mudança de processo), de modo que a investigação de processo e a ação corretiva possam ser realizadas antes que muitas unidades sejam fabricadas. Nessa mesma perspectiva Prajapati e Mahapatra (2008) mostram que o objetivo do CEP é monitorar os processos, identificar causas especiais de variação e sinalizar para uma tomada de decisão correta, quando for apropriado. Conforme Ghinato (1998) o que inibe a maior utilização 
do CEP é o fato de modelos estatísticos desenvolvidos focarem o princípio matemático e não a resolução do problema no chão de fábrica;

- o Sistema de Inspeção Sucessiva, que conforme Ghinato (1996), essa modalidade surgiu da necessidade de inspeção $100 \%$ e da necessidade de atuação proativa e rápida em caso de constatação de defeito. Esse tipo de inspeção é estendido a todas as estações de trabalho, de forma que cada trabalhador inspecione o item recebido da etapa anterior antes de executar sua operação. Os pontos positivos desse tipo de inspeção são os fatos de que o índice de defeitos por falta de atenção é minimizado, as etapas anteriores estão vinculadas a etapa seguinte e a inspeção é conduzida por pessoas independentes aos processos;

- o Sistema de Auto-Inspeção (SAl), é considerado o sistema mais eficaz de inspeção informativa, visto que a inspeção é realizada pelo operador responsável pelo processamento, possibilitando ação corretiva instantânea. Além disso, outro fato que contribui é o de que as pessoas preferem descobrir os seus problemas, ao invés desses serem apontados por terceiros (SHINGO, 1996). Todavia, a maior limitação da SAI é o foco na detecção de defeitos ao invés da detecção de erros no processamento. A principal diferença entre o CEP e as outras técnicas está na forma de inspeção. O CEP é realizado por amostragem sobre variáveis e atributos. Entretanto, a SAI e a SIS são inspeções $100 \%$ realizadas sobre variáveis e atributos.

c) a Inspeção na Fonte é definida por Shingo (1988) como a mais eficiente, visto que seu objetivo é atuar preventivamente e eliminar defeitos. Denominada em processos de manufatura como "controle adaptável" (BLACK, 1998), compensa ou corrige a condição de erro para prevenir a fabricação de um item defeituoso. A principal vantagem da inspeção na fonte demonstrada nos estudos de Takasan (1992) está no ciclo de controle mais curto em relação aos outros métodos de inspeção. Nesse 
método, o erro acontece e é detectado instantaneamente, a causa do erro é verificada e a ação corretiva é implantada. Dessa forma a atuação se dá sobre o processamento e não sobre o produto, o que viabiliza o zero defeito. Apesar dessa segurança, Shingo (1988) ressalta que se deve verificar o impacto do processo sobre os demais processamentos, a fim de evitar uma inspeção na fonte que seja desnecessária. Por exemplo, não é necessário que sejam instituídos processos de inspeção na fonte para operações de montagem do produto, mas nas operações de fabricação das partes desse mesmo produto.

\subsection{Conceito de poka-yoke}

Os poka-yokes são aplicados em diversos contextos (logística, saúde, construção civil, tecnologia da informação), não necessariamente associados a iniciativas de implantação da produção enxuta. Contudo, nem sempre estes contextos são coincidentes, complementares, ou evoluem durante os anos, conforme se observa no quadro 1.

No quadro 1, observam-se as principais características conceituais de sistemas poka-yokes que são comuns a 19 conceitos distintos. Em $73 \%$ os conceitos de poka-yoke mencionam a prevenção de defeitos ou detecção de erros, sem realizar uma diferenciação entre os conceitos de erros e defeitos. A diferenciação é importante, visto que permite classificar a função dos poka-yokes como sendo reativa (protetora) ou pró-ativa (preventiva). Conforme definição de Shingo (1996), o defeito é um dano ocorrido ao projeto de produção, seja ele um produto ou serviço. De outro lado, um erro pode ser entendido como uma falha no planejamento ou execução de uma operação (REASON, 1997), sendo normalmente a causa imediata dos defeitos. Assim, neste estudo considera-se que os poka-yokes com função reativa detectam defeitos, enquanto os poka-yokes com função pró-ativa detectam erros e, como resultado disso, previnem defeitos. 
Quadro 1 - Características de conceitos de sistemas poka-yoke

\begin{tabular}{|c|c|}
\hline Autor/Ano & Características conceituais comuns \\
\hline $\begin{array}{l}\text { Nikkan (1988), Bendel et al. (1995), } \\
\text { Moores (1996), Ghinato (1996), Plonka } \\
\text { (1997), Fischer (1999), Patel et al. } \\
\text { (2001a), Patel et al. (2001b), Stewart e } \\
\text { Grout (2001), Formoso et al. (2002), } \\
\text { McGee (2005). }\end{array}$ & $\begin{array}{l}\text { Os conceitos mostram que os poka-yokes são dispositivos } \\
\text { que detectam, eliminam e corrigem os erros, no mais alto } \\
\text { nível de controle sobre o processo produtivo. }\end{array}$ \\
\hline $\begin{array}{l}\text { Black (1998), Middleton (2001), Lean } \\
\text { Institute (2003), Conti et al. (2006). }\end{array}$ & $\begin{array}{l}\text { Os conceitos indicam que os poka-yokes são } \\
\text { procedimentos, métodos, técnicas usadas para eliminar o } \\
\text { julgamento ou displicência de operação e produzir com alta } \\
\text { confiabilidade. }\end{array}$ \\
\hline $\begin{array}{l}\text { Santos e Powell (1999), Connor } \\
\text { (2006), Hinckley (2007). }\end{array}$ & $\begin{array}{l}\text { Os conceitos revelam que os poka-yokes são sistemas que } \\
\text { garantem à concepção do produto de forma óbvia, } \\
\text { detectando ou prevenindo a ocorrência de defeitos. }\end{array}$ \\
\hline $\begin{array}{l}\text { Nikkan (1988), Bendel et al. (1995), } \\
\text { Moores (1996), Ghinato (1996), Plonka } \\
\text { (1997), Black (1998), Fischer (1999), } \\
\text { Santos e Powell (1999), Middleton } \\
\text { (2001), Patel et al. (2001a), Patel et al. } \\
\text { (2001b), Stewart e Grout (2001), } \\
\text { Formoso et al. (2002), Lean Institute } \\
\text { (2003), McGee (2005), Conti et al. } \\
\text { (2006), Connor (2006), Hinckley } \\
\text { (2007), Grout (2007). }\end{array}$ & $\begin{array}{l}\text { Os conceitos diferenciam a função reativa (detecção do } \\
\text { erro ou defeito) da função pró-ativa (prevenção do erro ou } \\
\text { defeito) dos poka-yokes. }\end{array}$ \\
\hline $\begin{array}{l}\text { Nikkan (1988), Bendel et al. (1995), } \\
\text { Moores (1996), Ghinato (1996), Plonka } \\
\text { (1997), Black (1998), Fischer (1999), } \\
\text { Santos e Powell (1999), Middleton } \\
\text { (2001), Patel et al. (2001a), Patel et al. } \\
\text { (2001b), Stewart e Grout (2001), } \\
\text { Formoso et al. (2002), Lean Institute } \\
\text { (2003), McGee (2005), Conti et al. } \\
\text { (2006). }\end{array}$ & $\begin{array}{l}\text { Os conceitos restringem a utilização de poka-yokes a } \\
\text { sistemas de manufatura. }\end{array}$ \\
\hline $\begin{array}{l}\text { Santos e Powell (1999), McGee } \\
\text { (2005), Hinckley (2007). }\end{array}$ & $\begin{array}{l}\text { Os conceitos associam a utilização de sistemas poka- } \\
\text { yokes à estabilidade da produção. }\end{array}$ \\
\hline $\begin{array}{l}\text { Connor (2006), Hinckley (2007), Grout } \\
\text { (2007). }\end{array}$ & $\begin{array}{l}\text { Os conceitos aplicam o idéia de poka-yokes a áreas } \\
\text { diferentes da manufatura como as áreas de saúde, } \\
\text { tecnologia da informação e atividades domésticas. }\end{array}$ \\
\hline
\end{tabular}

Fonte: Elaborado pelos autores 
Observa-se também, no quadro 1 , que com relação à classificação dos poka-yokes, $63 \%$ dos estudos classificam os mesmos como dispositivos, $21 \%$ como procedimentos, métodos e técnicas, e $16 \%$ como sistemas. Neste estudo, a perspectiva adotada é de que os poka-yokes são sistemas e devem ser desenvolvidos segundo um método que considere todo o seu ciclo de vida, desde a decisão de usar ou não um poka-yoke até a sua descontinuidade de uso. De fato, é necessário um sistema de gestão para poka-yoke (SGPK), possibilitando o projeto, operação, manutenção e descontinuidade do poka-yoke.

$\mathrm{O}$ quadro 1 também revela quais os setores que os autores tinham em mente quando definiram o conceito de poka-yoke. Segundo esse critério, em 16\% dos casos os autores visam aplicações nas áreas da saúde, tecnologia da informação, ou mesmo nas atividades domésticas, extrapolando a limitação de manufatura imposta pelos demais $84 \%$ de definições.

Além disso, em 100\% das definições não há uma diferenciação entre os conceitos de prevenção e detecção de defeitos. Observa-se que 37\% destes conceitos associam implicitamente o poka-yoke a função pró-ativa (prevenção do erro e do defeito), contudo nessas mesmas definições a função reativa também é ressaltada.

Observa-se também que em $16 \%$ das definições há uma associação entre o conceito do poka-yoke e o conceito da estabilidade da produção. Nesses conceitos o poka-yoke é mostrado como um mecanismo de garantia da estabilidade, atuando sobre a disponibilidade de recursos para o sistema de manufatura.

Essas análises subsidiam a proposição de um conceito de poka-yoke a ser usado neste trabalho. Portanto, nesse estudo os poka-yokes são definidos como sistemas destinados à prevenção e detecção de perdas de qualquer natureza (por exemplo, produtos defeituosos e acidentes de trabalho), sendo constituídos por barreiras físicas e/ou funcionais e/ou simbólicas, que contribuem para a redução da variabilidade e manutenção da estabilidade em processos. Barreiras físicas são aquelas que não permitem o transporte de massa, energia ou informação, bem como não dependem da interpretação do usuário (por exemplo, um muro). Barreiras funcionais estabelecem pré-condições que devem ser atendidas antes que um evento ocorra (por exemplo, uma senha). Barreiras simbólicas requerem 
interpretação, percepção e resposta do usuário (por exemplo, um cartaz) (HOLLNAGEL, 2004).

\subsection{Classificações de poka-yoke}

As aplicações práticas de sistemas poka-yokes com frequência consistem de gabaritos, sensores e alarmes. Por exemplo, Shimbun (1988) apresenta uma relação de 240 poka-yokes observados em 100 indústrias diferentes, incluindo as áreas de eletrônicos, automóveis e indústria pesada. Similarmente, Grout (2007) apresenta um manual com poka-yokes que poderiam ser desenvolvidos e aplicados na área da saúde.

Contudo, é possível estabelecer categorias analíticas de poka-yokes que abstraiam seus princípios operacionais e diferenciem dispositivos que, embora usem os mesmos mecanismos físicos, possuem propriedades distintas. Uma dessas categorias diz respeito a já citada diferenciação entre poka-yokes pró-ativos e reativos.

Outra classificação relativamente conhecida é a proposta por Shingo (1988), que classifica os sistemas poka-yokes de acordo com o objetivo e as técnicas utilizadas. Quando vinculados ao objetivo, referem-se à função de regulagem, e quando ligados as técnicas referem-se à função de detecção.

A classificação de Shingo (1988) divide a função de regulagem em método do controle e método da advertência. O método do controle é assim denominado, pois o poka-yoke detecta uma variabilidade não esperada no processo e interrompe a operação, com os objetivos de evitar a produção de defeitos em série e criar um senso de urgência para que a ação corretiva seja implementada. Outra característica do método do controle é que o operador não possui graus de liberdade para tomada de decisão, sendo induzido a realizar a ação correta. No método da advertência, o poka-yoke detecta a anormalidade, mas não interrompe o processo, apenas sinalizando a ocorrência através de sinais sonoros e/ou visuais.

Já a função de detecção é dividida em método do contato, método do conjunto e método das etapas. O método do contato aplica-se tipicamente para 
detectar anormalidades nas dimensões, através de dispositivos que se mantêm em contato com o produto. O método do conjunto é utilizado em operações executadas em uma sequência de movimentos ou etapas idênticas, garantindo que nenhum desses passos seja negligenciado. O método das etapas também é usado para garantir que nenhuma operação seja negligenciada. Contudo, diferentemente do método do conjunto, no método das etapas as operações sequenciais não são idênticas.

Em particular, vale reforçar as oportunidades para uso integrado de pokayoke e CEP. Ghinato (1996) ressalta que erroneamente a aplicação de poka-yoke costuma ser restrita a processos sem um forte controle estatístico. Contudo, os processos que são controlados estatisticamente são os que apresentam as maiores e melhores oportunidades de aplicação de poka-yokes, visto que as cartas de controles estatísticos geram as informações que subsidiam a escolha das categorias de poka-yokes mais apropriados. Por exemplo, em um processo de pesagem de matéria-prima para a fabricação de pastilhas de freio. O controle de peso $(\mathrm{kg}) \mathrm{de}$ matérias-primas é usado para atender uma quantidade de peças por lote. Normalmente o operador não realiza uma pesagem adequada pela necessidade de atender múltiplos processos de pesagem durante o turno de trabalho. Valores de peso são controlados por amostragem a cada cinco pesagens analisadas e registrados de um gráfico de controle. A análise dessa carta de controle mostra que com passar do tempo o valor de pesagem tende do valor nominal para a parte inferior, até extrapolar o limite inferior de controle. Nesse caso a implementação do poka-yoke que garantisse o valor $(\mathrm{kg})$ de pesagem, não permitindo que o operador removesse a matéria-prima da balança, até o valor nominal estabelecido, garantiria que $\mathrm{o}$ atendimento de toda a matéria-prima necessária para a confecção do lote. Esse caso ilustra a percepção de Ghinato (1998) sobre a relação existe entre o desenvolvimento dos sistemas poka-yokes e os gráficos para controle de qualidade. 


\subsection{Métodos para elaboração de poka-yoke}

Shingo (1988) propõe um conjunto de etapas para a elaboração dos pokayokes. Na primeira, deve-se implantar um método de CEP para verificar onde os defeitos e problemas ocorrem freqüentemente. $\mathrm{Na}$ sequência, inicia-se o desenvolvimento dos poka-yokes que visem a controlar as causas mais freqüentes de defeitos. Esses projetos são concebidos pela equipe de trabalho designada para implantação de poka-yokes. A seguir, ocorre a implantação do poka-yoke e também a implantação de pontos de inspeção após o ponto de uso do poka-yoke, a fim de medir sua eficácia. Gradualmente, os pontos de inspeção são eliminados, até chegar-se a ocorrência de zero defeitos e a estabilidade do processo. Quando esse estado é obtido, o poka-yoke é estendido a processos similares.

McGee (2005) propôs cinco etapas: (a) identificar o defeito e o impacto desse defeito sobre o cliente; (b) identificar em que etapa do processo o defeito foi descoberto, para posteriormente descobrir em qual etapa ele foi criado; (c) identificar a causa raiz que originou o defeito; (d) realizar um brainstorming com a equipe de trabalho para detectar formas de eliminar os desvios de processo; (e) criar, testar, validar e implantar o dispositivo poka-yoke.

Para Connor (2006) a implantação do poka-yoke está vinculada à implantação da filosofia kaizen. A filosofia kaizen tem por princípio a melhoria contínua, e o autor defende que o poka-yoke deve ser continuamente melhorado, a fim de suprir as alterações ocorridas no processo. Dessa forma, o primeiro passo é documentar as variáveis do processo, com a identificação de potenciais defeitos em cada passo do processo. A identificação dos potenciais defeitos é realizada através de uma série de questões, conduzidas e documentadas pelos membros da equipe. Em seguida, o método de Connor (2006) propõe que sejam priorizados alguns desses potenciais defeitos e sejam realizadas observações nos processos que os originam. A partir disto, a equipe realiza um brainstorming sobre possíveis dispositivos poka-yokes ou outras técnicas a serem aplicadas para a prevenção do defeito. $\mathrm{Na}$ seqüência, aplica-se o poka-yoke e acompanha-se o seu desenvolvimento no processo. 
Para Hinckley (2007) o processo de desenvolvimento de um poka-yoke deve estar baseado no ciclo de solução de problemas PDCA (Plan - Do - Check Action), envolvendo as seguintes etapas: (a) identificar o problema; (b) analisar o problema; (c) gerar soluções potenciais; (d) selecionar e planejar a implantação das soluções; (e) implementar as soluções; (f) avaliar as soluções. Nesta última etapa, quando a avaliação for positiva deve ser padronizada e implantada em todos os processos similares. Quando o poka-yoke é validado, deve-se padronizar essa solução para todos os problemas semelhantes existentes na organização. Hinckley (2007) detalha a fase de identificação de problemas em subfases: (a) identificar com que freqüência o problema ocorre; (b) avaliar o impacto do problema no fluxo de processo; (c) avaliar o impacto do problema sobre o cliente final.

Uma constatação realizada no estudo de Hinckley (2007) é de que o problema causado por erros humanos necessita de classificação pelo tipo de erro. De fato, diferentes tipos de erros humanos implicam em medidas preventivas com diferentes ênfases. Os poka-yokes são fortemente indicados para detectar erros que ocorrem durante atividades rotineiras, em comportamentos automatizados dos operadores. Nessas situações os operadores não estão com a atenção focada na tarefa e, por definição, os poka-yokes funcionam independentemente da atenção do operador (SAURIN et al., 2007).

No método de Grout (2007) existem oito etapas para o desenvolvimento de poka-yokes. A primeira etapa envolve selecionar um modo de falha para análise, dentre todos os modos de falha identificados no processo. Nesse passo, a ferramenta utilizada é o Failure Mode and Effect Analysis (FMEA). No segundo passo realiza-se um brainstorming para indicar soluções para controle os modos de falha. Com a revisão do FMEA, diversos princípios dos modos de falha serão identificados, o que permite que no terceiro passo seja desenvolvida uma árvore detalhada de modos de falhas indesejáveis. A partir dessa árvore, no quarto passo, é possível identificar o modo de falha raiz que desencadeia os demais modos de falha indesejados. No quinto passo, identificam-se recursos que podem ser usados para controlar o modo de falha raiz, que gera, no sexto passo, uma primeira alternativa de projeto de dispositivo a prova de erros. No sétimo passo, devem ser identificadas outras alternativas de solução desenvolvidas no quarto passo, visto que 
elas podem apontar para falhas que não estão sendo cobertas pelo princípio de solução estabelecido; essa etapa é cíclica, até que ocorra a identificação do princípio de solução ideal. No oitavo passo é realizada a implementação da solução final.

O estudo de Grout (2007) apresenta também diretrizes para a etapa de implementação dos poka-yokes. Segundo aquele autor, deve-se considerar que a solução adotada pode enfrentar resistências organizacionais (por exemplo, a solução encontrada pode eliminar postos de trabalho em operações, gerando resistência por medo em operadores), e pode possuir altos custos de implantação. Grout (2007) sugere o desenvolvimento de um protótipo, antes de generalizar a implementação a todos os processos.

Embora os estudos citados neste item apresentem diretrizes para a elaboração de poka-yokes, nenhum deles constitui um método consolidado para a gestão de poka-yokes ao longo de todo o seu ciclo de vida. O quadro 2 sumariza as principais diretrizes identificadas nos estudos revisados. 
Quadro 2 - Técnicas relacionadas a poka-yokes

\begin{tabular}{|c|c|c|c|c|c|c|}
\hline \multirow[b]{2}{*}{ Atividades desenvolvidas } & \multicolumn{5}{|c|}{ Autor } & \multirow{2}{*}{$\begin{array}{l}\text { Número } \\
\text { de } \\
\text { incidênci } \\
\text { as }\end{array}$} \\
\hline & $\begin{array}{c}\text { Shing } \\
0 \\
(1988)\end{array}$ & $\begin{array}{l}\text { McGe } \\
\text { e } \\
(2005)\end{array}$ & $\begin{array}{c}\text { Conno } \\
r \\
(2006)\end{array}$ & $\begin{array}{l}\text { Hinckle } \\
\text { y (2007) }\end{array}$ & $\begin{array}{l}\text { Grout } \\
(2007 \\
\quad)\end{array}$ & \\
\hline $\begin{array}{l}\text { Utilizar equipe multidisciplinar de } \\
\text { desenvolvimento. }\end{array}$ & $x$ & $x$ & $\mathrm{x}$ & $\mathrm{X}$ & $x$ & 5 \\
\hline $\begin{array}{l}\text { Implantar poka-yoke e acompanhar } \\
\text { seu desenvolvimento no processo. }\end{array}$ & $\mathrm{X}$ & $\mathrm{X}$ & $\mathrm{X}$ & $\mathrm{X}$ & $x$ & 5 \\
\hline $\begin{array}{l}\text { Buscar princípios de solução, para os } \\
\text { problemas verificados, com a equipe } \\
\text { de desenvolvimento, e documentar } \\
\text { esses princípios. }\end{array}$ & & $x$ & $\mathrm{x}$ & $x$ & $x$ & 4 \\
\hline $\begin{array}{l}\text { Determinar qual a causa raiz do } \\
\text { problema. }\end{array}$ & & $x$ & & $x$ & $x$ & 3 \\
\hline $\begin{array}{l}\text { Desenvolver projeto inicial para } \\
\text { poka-yoke. }\end{array}$ & $\mathrm{X}$ & $\mathrm{X}$ & & & $\mathrm{X}$ & 3 \\
\hline 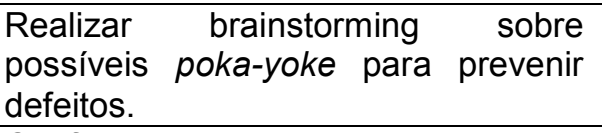 & & $X$ & $X$ & & $\mathrm{X}$ & 3 \\
\hline $\begin{array}{l}\text { Conferir os outros princípios de } \\
\text { solução, a fim de identificar falhas } \\
\text { não cobertas pelo princípio de } \\
\text { solução ideal estabelecido. }\end{array}$ & & & & $x$ & $x$ & 2 \\
\hline $\begin{array}{l}\text { Generalizar poka-yoke para todos os } \\
\text { processos e operações similares ao } \\
\text { poka-yoke implantado. }\end{array}$ & $\mathrm{X}$ & & $X$ & & & 2 \\
\hline $\begin{array}{l}\text { Aprovar poka-yoke no processo } \\
\text { produtivo. }\end{array}$ & $\mathrm{X}$ & & & $\mathrm{X}$ & & 2 \\
\hline $\begin{array}{l}\text { Implantar pontos de inspeção e } \\
\text { medição de eficácia. }\end{array}$ & $x$ & & & $x$ & & 2 \\
\hline $\begin{array}{l}\text { Identificar erro e seu impacto sobre o } \\
\text { cliente final. }\end{array}$ & & $\mathrm{X}$ & & $\mathrm{X}$ & & 2 \\
\hline $\begin{array}{l}\text { Documentar as variáveis do } \\
\text { processo e identificar pontos de erros } \\
\text { potenciais. }\end{array}$ & & & $X$ & & & 1 \\
\hline $\begin{array}{l}\text { Analisar dados do controle estatístico } \\
\text { do processo. }\end{array}$ & $x$ & & & & & 1 \\
\hline Priorizar os potenciais defeitos & & & $\mathrm{x}$ & & & 1 \\
\hline $\begin{array}{l}\text { Determinar a frequência } \\
\text { ocorrência do problema. }\end{array}$ & & & & $\mathrm{X}$ & & 1 \\
\hline Avaliar as soluções implementadas. & & & & $\bar{X}$ & & 1 \\
\hline $\begin{array}{l}\text { Identificar recursos que podem ser } \\
\text { usados para solução de falha uma } \\
\text { indesejada. }\end{array}$ & & & & & $X$ & 1 \\
\hline
\end{tabular}

Fonte: Elaborado pelos autores 
Observa-se que todas as diretrizes propõem a participação de uma equipe de desenvolvimento para os poka-yokes. Também em todos os casos as diretrizes priorizam soluções da "causa raiz do defeito" (GROUT, 2007). Isto é importante, visto que permite a uma organização focar na criação de uma solução genérica para o problema.

As diretrizes existentes contemplam, como limite do ciclo de vida dos pokayokes, a fase de implementação. Contudo, essa visão deveria ser ampliada uma vez que o contexto que gerou a necessidade do poka-yoke pode mudar, tornando-o obsoleto. As mudanças nos processos podem ocorrer decorrentes de variabilidades, que implicam em novas oportunidades de erros.

A necessidade de manutenção do poka-yoke também não está explícita nas diretrizes apresentadas no quadro 2. De fato, deve haver manutenções preventivas que permitam que o poka-yoke continue desempenhando suas funções. $O$ uso dos poka-yokes como elementos de uma estratégia de gerenciamento visual também não está explícito nas diretrizes. Todavia, os poka-yokes dão visibilidade aos erros e defeitos, sendo necessárias estruturas de apoio que garantam a existência de recursos para solução o mais breve possível dos problemas. Por exemplo, quando os poka-yokes são usados em sistemas de produção enxuta, pode ser projetada uma estrutura de cadeia de ajuda, que padroniza quem são os responsáveis por prestar apoio aos operadores que detectaram os erros ou defeitos (KAMADA, 2007).

\subsection{Relações entre poka-yokes e tipos de inspeção}

A funcionalidade principal de combinar poka-yokes e operações de inspeção está na redução substancial dos erros e defeitos nos processos. Conforme Shingo (1996) quando combinados esses mecanismos conduzem ao controle de qualidade zero defeito (CQZD) em processos.

Os tipos de erros combatidos pela combinação desses mecanismos são definidos nesse trabalho pelos desvios em relação ao método de execução correto, sendo que aqueles que executavam a tarefa tinham os recursos disponíveis para executar o método correto; e houve uma tomada de decisão incorreta, sendo que os 
recursos para a tomada de decisão correta estavam disponíveis. Conforme Reason (1997) esses erros podem estar associados a habilidades, regras e conhecimento.

Erros no nível da habilidade (skill-based errors, SB) são aqueles que o operador realiza comportamentos automáticos e rotineiros, com baixo nível de consciência. Os erros tipicamente envolvem falhas de execução, sendo que os lapsos e deslizes são as mais comuns. Erros no nível das regras (rule-based errors, RB) são aqueles que o operador aumenta a consciência para aplicar regras familiares em desvios também familiares das situações rotineiras. Erros no nível do conhecimento (knowledge-based errors, KB) são aqueles que o operador atua em alto nível de consciência para resolver problemas que não dispõem de regras. Os erros são bastante prováveis quando o operador é requisitado a operar nesse nível, dentre outros motivos pelo fato de que normalmente há pressões organizacionais que limitam o tempo e os recursos para a tomada de decisão.

Esses tipos de erros poderiam ser inibidos, ou eliminados, pela combinação de poka-yokes e operações de inspeção. Todavia, não é recomendável a combinação de poka-yokes a todos os tipos de inspeção. Por exemplo, inspeções na fonte devem ser priorizadas na combinação com poka-yokes, em relação a inspeções por julgamento, visto que no julgamento a perda já está consumada e na fonte a perda foi evitada.

Uma combinação em que os tipos de erros poderiam ser minimizados é a elaboração de poka-yoke através da inspeção informativa, utilizando o CEP (Controle Estatístico do Processo). O CEP forneceria causas de anormalidades do processo. Salienta-se que anormalidades de processo são provenientes de todos os fatores de instabilidades causadas por erros ou defeitos (GHINATO, 1996). Nesse caso o sistema poka-yoke não dispensa a inspeção, mas a utiliza como base para seu projeto.

\subsection{Papel dos poka-yokes na estabilidade da produção}

Conforme Liker e Meier (2007) a estabilidade de produção pode ser definida como a capacidade de produzir resultados coerentes ao longo do tempo. Já Kamada (2007) entende que a estabilidade ocorre quando se consegue produzir de acordo 
com o planejado, com o menor desperdício possível, sem afetar a segurança e garantindo a qualidade. Além disso, a estabilidade da produção para Liker e Meier (2007) é atingida através da combinação de mão-de-obra, método, materiais e máquinas. Portanto, a garantia da estabilidade na produção passa pelo combate às perdas. Essa perspectiva permite concluir que mecanismos de controle de qualidade, como poka-yokes e operações de inspeção, contribuem à garantia da estabilidade, seja de forma reativa (detectar erros) ou pró-ativa (prevenir erros). Exemplos, como ausência de manutenção preventiva e falta de padronização, descritos por Liker e Meier (2007), fortalecem a vinculação existente entre a estabilidade na produção e os mecanismos de controle de qualidade.

Outra forma de tratar a estabilidade é através da análise estatística do processo. Sob esta perspectiva, Montgomery (2004), define que um processo está estável quando permite a redução sistemática da variabilidade nas característicaschave do produto, fornecendo as ferramentas necessárias para avaliação e melhoria de processos, produtos e serviços de forma robusta e abrangente. Complementarmente, o autor exemplifica através de três fatores as causas para variabilidade de processo: máquinas ajustadas ou controladas de maneira inadequada, erros do operador e matérias-primas defeituosas.

Essas exemplificações permitem identificar evidências objetivas de instabilidade na produção, como: (a) alto grau de variação nas medidas de desempenho; (b) motivos para mudanças frequentes de planos (VANDERLEl et al, 2009); (c) ausência de método de trabalho padrão; (d) variabilidade de estoque em processo; (e) independência entre operações, fluxo inconsistente ou inexistente (NOGUEIRA E SAURIN, 2008); (f) autonomia para funcionários que não estão suficientemente treinados em operações complexas (PONTES e ZANAROTTI, 2007). Nestes exemplos, sistemas poka-yokes funcionariam como agentes estabilizadores, atuando sobre as evidências de instabilidades.

Dessa forma, a garantia da estabilidade é suportada pelos mecanismos de controle de qualidade, como operações de inspeção e poka-yokes, discutidos anteriormente nesse artigo. A garantia da estabilidade através sistemas poka-yokes é uma abordagem desenvolvida por Shingo (1988). O autor relata a implementação de poka-yokes decorrentes instabilidade observadas em cartas de controle 
estatístico. O CEP, por exemplo, pode indicar onde os poka-yokes podem ser implantados, uma vez que aponta claramente as causas aleatórias nos processos. Essas causas são em muitas vezes repetitivas, ocorrendo em situações claramente identificáveis, tais como trocas de turno, retomada de jornada, operações de setup, entre outras. Além disso, os processos onde os procedimentos estatísticos para o CEP revelam-se complexos podem ser também controlados por poka-yoke, como por exemplo, fabricação de matrizes e moldes, construção de software, aplicação de medicamentos em pacientes.

A vinculação de poka-yokes para a garantia da estabilidade através da estabilidade de recursos ainda é um campo de estudo em aberto. Na revisão realizada não foram encontradas evidências dessa vinculação. Todavia, pode-se afirmar que o poka-yoke assegura que o erro ou defeito não ocorra garantindo a disponibilidade do recurso.

\section{CONSIDERAÇÕES FINAIS}

Este artigo ilustrou a diversidade de conceitos de poka-yokes, bem como de diretrizes para seu desenvolvimento. A verificação de conceitos e diretrizes relacionados a poka-yokes identifica a inexistência de padronizações para identificação das causas de problemas que levam ao projeto do sistema poka-yoke, a ausência de indicadores de eficiência e eficácia de poka-yokes, a ausência de princípios de gestão visual, a desconsideração das fases de manutenção e descontinuidade no ciclo de vida do poka-yoke.

Em relação aos conceitos de poka-yoke, a conclusão foi de que existe uma diversidade de posições à respeito do tema. Em particular, ficou claro que os pokayokes podem ser de diversos tipos, mecanismos de funcionamento e grau de eficácia. A literatura não esclarece como identificar a configuração mais adequada do poka-yoke para cada tipo de perda e inspeção em que eles podem ser aplicáveis.

Em relação às diretrizes, a conclusão foi de que há uma preocupação voltada para o projeto e concepção de poka-yokes até a sua implementação, sendo negligenciadas a manutenção e descontinuidade de uso do poka-yoke. Assim, esse estudo indicou ser necessária a existência de sistemas de gestão de poka-yokes 
(SGPK) que contemplem todo o ciclo de vida. Além disso, os SGPK devem estar baseados no ciclo de melhoria contínua, o PDCA. As modificações que ocorrem em processos alteram sua estabilidade e o PDCA garantiria a estabilidade para um SGPK. O ciclo de melhoria contínua permitiria constantes verificações do poka-yoke, de sua adequabilidade ao sistema de manufatura e também da possibilidade de reaproveitar esse sistema. Resumidamente PDCA possibilitaria o controle de projeto, operação, manutenção e descontinuidade de uso dos poka-yokes.

A utilização de técnicas da qualidade para desenvolvimento de sistemas poka-yokes também é um campo de estudo em aberto. Por exemplo, o FMEA pode ser útil na etapa de identificação das causas de instabilidade, ou então com a elaboração do princípio de soluções a partir de múltiplas soluções verificadas, como mostram os estudos de Grout (2007).

Outra oportunidade de pesquisa diz respeito à investigação do vínculo entre - CEP e os sistemas poka-yokes. O CEP como sistema de inspeção pode ser uma das principais fontes de informação para projetos de poka-yoke e um método para elaboração de poka-yoke pode ser apoiado por essa ferramenta. Vinculações desse tipo permitiriam que a variabilidade não inerente do processo fosse tratada pela aplicação de sistemas poka-yokes, evitando a ocorrência de perdas, que são apenas indicadas em um sistema de CEP.

Quanto ao objetivo do trabalho de mostrar a diversidade de conceitos e nomenclaturas associados aos poka-yokes verifica-se que foi realizado, inclusive foi proposto um novo conceito para esses sistemas. O estudo dos conceitos de pokayoke gerou a necessidade de detalhar os tipos de métodos existentes para concepção desses sistemas. Esse detalhamento foi importante, visto que proporcionou o entendimento de quais são as diretrizes utilizadas no projeto de poka-yokes.

Uma questão que merece investigação é em relação à classificação dos poka-yokes como ferramentas que, combinadas as operações de inspeção na fonte, garantem o zero defeito. Essa lógica contribui para que o conceito de poka-yoke seja confundido a uma barreira, ou um dispositivo, quando na verdade o artigo mostrou que deve ser entendido como um sistema. Portanto, uma pesquisa para detalhar a 
diferença entre os sistemas poka-yokes e o seu papel quanto à obtenção de zero defeito é necessária.

A estabilidade de processos e recursos é dependente de uma gestão dos sistemas, ferramentas e metodologias que propõem melhoria contínua. Portanto, um SGPK capaz de englobar todas as fases do desenvolvimento medir o impacto de um poka-yoke sobre um sistema de manufatura. Para tanto, é necessário que novas pesquisas indiquem diretrizes para forma de gestão de sistemas poka-yokes.

Verifica-se que o estudo de poka-yokes é uma questão em aberto que necessita de pesquisas que, primeiramente focalizem a definição conceitual dos sistemas poka-yokes, e, posteriormente, a elaboração de diretrizes que remetam a técnicas consistentes de elaboração e gestão de poka-yokes.

\section{REFERÊNCIAS}

BENDELL, T., PENSON, R., CARR, S. The quality gurus: their approaches described and considered. Managing Service Quality, v.5, n.6, p. 44-48, 1995.

BLACK, J. T. O projeto da fábrica com futuro. Porto Alegre: Artes Médicas Sul Ltda, 1998.

CONNOR, G. Poka-yoke: Human-Proof your Process. Journal of Industrial Maintenance e Plant Operations, p.12-14, jun. 2006.

CONTI, R., ANGELIS, J., COOPER, C., FARAGHER, B., GILL, C. The effects of lean production on worker job stress. International Journal of Operations and

Production Management, v.26, n.9, p.1013-1038, 2006.

CROSBY, P. B. Qualidade é investimento: a arte de garantir a qualidade. Rio de Janeiro: José Olímpio S.A., 1999.

DEMING, W. E. Qualidade: a revolução da administração. Rio de Janeiro: Marques Saraiva S.A., 1990.

DIAS, J. R., INFANTE, P. Controls Charts with predetermined sampling intervals. International Journal of Quality and Reliability Management, v.25, n.4, p.423435, 2008.

FEIGENBAUM, A. V. Controle de qualidade total gestão e sistemas. São Paulo: McGraw-Hill Ltda, 1994. 
FISHER, M. Process improvement by poka-yoke. Work Study MCB University Press, v.48, n.7, p. 264-266, 1999.

FORMOSO, C. T., SANTOS, A., POWELL, J. A. An exploratory study on the applicability of process transparency in construction sites. Journal of Construction Research, v.3, n.1, p.35-54, 2002

GARVIN, D. A. Gerenciando a qualidade: a visão estratégica e competitiva. Rio de Janeiro: Qualitymark, 1992.

GHINATO, P. O Sistema toyota de produção: mais do que simplesmente o just-intime. Caxias do Sul, Editora da UCS, 1996.

GHINATO, P. Quality control methods: toward modern approaches through well estabilished principles. Total Quality Management Journal, v.9, n.6, dez. 1998.

GROUT, J. Mistake-proofing the design of the health care processes. Rockville, AHRQ, 2007.

HINCKLEY, C. M. Combining mistake-proofing and Jidoka to achieve world class quality in clinical chemistry. General Paper. Springer-Verlag, v.12 p. 223-230, mar. 2007.

HIRATA, H. S. Sobre o "modelo" japonês: automatização, novas formas de organização e de relações de trabalho. São Paulo, Editora da USP, 1993.

HOLLNAGEL, E. Barrier analysis and accident prevention: how to improve safety by understanding the nature of accidents rather than finding their causes, 2004. 37p.

INSTITUTO LEAN. Glossário para praticante do pensamento lean. Lean Institute Brasil, São Paulo, 2003. (Apostila).

ISHIKAWA, K. Controle de qualidade total: à maneira japonesa. Rio de Janeiro: Campus Ltda, 1993.

JURAN, J. M., GRYNA, F.M. Controle da qualidade: conceitos, políticas e filosofia da qualidade. São Paulo: McGraw-Hill Ltda, 1991.

LIKER, K. J., MEIER, D. O Modelo toyota de produção: manual de aplicação. Porto Alegre: Bookman, 2007. 432 p.

KAMADA, S. Estabilidade de produção da toyota do Brasil.São Paulo: Lean Institute Brasil, 2007. Disponível em:

<http://www.lean.org.br/bases.php?\&interno=artigo 44> Acesso em: 27 jul. 2008.

MCGEE, D. Lean and Six Sigma: A Holistic Approach to Process Improvement. In.: ASQ- AMERICAN SOCIETY FOR QUALITY CONGRESS, Proceedings... Denver, USA, nov. 2005. 
MIDDLETON, P. Lean software development: two case studies. Software Quality Journal, v.9, p.241-252, 2001.

MONTGOMERY, D. C. Introdução ao controle estatístico da qualidade. 4.ed. Rio e Janeiro: LTC, 2004.

MOORES, B. A glossary of terms encountered in quality and customer service. International Journal of Health Quality Assurance, v.9, n.5, p. 24-36, 1996.

NIKKAN. K. S. Poka-yoke: Improving product quality by preventing defects. Portland: Productivity Press, 1988.

NOGUEIRA, M. G. S. SAURIN, T. A. Proposta de avaliação do nível de implementação de típicas práticas de produção enxuta em uma empresa do setor metal-mecânico. Revista Produção On Line. Florianópolis, v.8, n. 2, jul. 2008. Disponível em: <http://producaoonline.org.br/index.php/rpo/article/view/115>. Acessado em: 2 nov. 2010.

PATEL, S., DALE, B. G., SHAW, P. Set-up time reduction and mistake proofing methods: an examination in precision component manufacturing. The TQM Magazine, v.13, n.3, p.175-179, 2001.

Set-up time reduction and mistake proofing methods: a study of application in small company. Journal of Business Process Management, v.7, n.1, p.65-75, 2001.

PLONKA, F. E. Developing a lean and agile work force. Journal of Human Factors and Ergonomics in Manufacturing, v.7, n.1, p.11-27, 1997.

PONTES, S. K., ZANAROTTI, V. R. C. Sistema de produção flexível e intensificação do trabalho: um ensaio teórico. Revista Produção On Line. Florianópolis, v.7, n.1, 2008. Disponível em: < http://producaoonline.org.br/index.php/rpo/article/view/100>. Acessado em: 2 nov. 2010.

PRAJAPATI, D. R., MAHAPATRA, P. B. A simple and effective $X$ chart for process monitoring. International Journal of Quality and Reliability Management, v.25, n.5, p.508-531, 2008.

REASON, J. Managing the risks of organizational accidents. England, Ashgate Publishing Limited, 1997. p.61-83.

SANTOS, A., POWELL, J. Potencial of poka-yoke devices to reduce variability in construction. Berkeley, University of California, p.51-62, 1999.

SAURIN, T. A., JACQUES, J., HENRIQSON, E., JÚNIOR, G. C. Análise de uma barreira de classificação contra acidentes em produtos e processos. XXVII ENCONTRO NACIONAL DE ENGENHARIA DE PRODUÇÃO. Anais... Foz do Iguaçu: ENEGEP, 2007. 
SHEWHART, W. A. Economic control of quality of manufactured product. New York: Van Nostrand, 1931.

SHIMBUN, N. K. Poka-yoke: improving product quality by preventing defects. Portland, MA: Productivity Press, 1988.

SHINGO, S. Zero quality control: source inspection and the poka-yoke system. Cambridge, MA: Productivity Press, 1988.

O Sistema toyota de produção: do ponto de vista da engenharia de produção. Porto Alegre: Artemed, 1996. 291p.

Sistema de troca rápida de ferramenta: uma revolução nos sistemas produtivos. Porto Alegre: Artemed, 2000. 327p.

STEWART, D. M., GROUT, J. R. The human side of mistake-proofing. Journal of Production and Operations Management, v.10, n.4, p.440-459, 2001.

TAKASAN, K. TPM: confiabilidade e otimização de equipamentos industriais. In: SEMINÁRIO INTERNACIONAL DE TPM, 1992, Tokyo. Anais... Tokyo: JIPM, 1992.

VANDERELEI, M. L., JUNIOR, J. M., MARINS, F. A. S., MIRANDA, G. W. A. Implantação de controle baseado no sistema de execução da manufatura (mes): análise em empresa de usinagem no setor aeronáutico. Revista On Line. Florianópolis, v.ix, n.iv, 2009. Disponível em: < http://producaoonline.org.br/index.php/rpo/article/view/380>. Acesso em: 2 nov. 2010.

WANG, W., ZHANG, W. Early defect identification: application of statistical process control methods. Journal of Quality in Maintenance Engineering, v.14, n.3, p.225236, 2008.

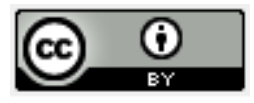

Artigo recebido em 05/04/2010 e aceito para publicação em 17/05/2011. 